\title{
漓江河岸带植物功能性状变异与关联
}

\author{
梁士楚 刘润红 荣春艳 常 斌 姜 勇* \\ 广西师范大学珍稀濒危动植物生态与环境保护教育部重点实验室/广西师范大学生命科学学院, 广西桂林 541006
}

\begin{abstract}
摘 要 研究植物功能性状随环境梯度的变异和关联格局, 对于认识不同环境梯度下群落构建和植物适应型具有重要意义。 该研究以漓江河岸带不同河段植物群落为研究对象, 调查了研究区内 36 个样方的物种组成, 测量了样方内 42 种木本植物的叶 面积 $(L A)$ 、比叶面积 $(S L A)$ 和木材密度 $(W D)$ 的功能性状值, 并运用性状梯度分析法对 3 个功能性状进行群落内( $\alpha$ 组分)和群落间 ( $\beta$ 组分)组分分解及相关性分析。结果表明: (1)群落平均 $L A$ 表现为中游最小且和下游差异显著, 群落平均 $W D$ 则表现为中上游 显著高于下游, 群落平均 $S L A$ 在两两河段间均差异显著。(2)不同河段的 3 个植物功能性状 $\beta$ 组分差异显著且实际观测值均小于 随机模拟的零模型分布, 但 $\alpha$ 组分在河岸带不同河段均差异不显著且 3 个功 能性状的 $\alpha$ 组分分布范围均小于 $\beta$ 组分, 说明在河 岸带不同河段的群落构建过程中环境笁选的作用要大于群落内种间的相互作用。(3)性状 $S L A$ 与 $L A$ 在群落间和群落内呈现出实 际观测和随机模拟的相关性均较低, 暗示了 $L A$ 和 $S L A$ 各自代表了植物在不同生态策略上的维度; 但 $S L A$ 和 $W D$ 实际观测值和 随机模拟值呈现出较强的负相关关系, 暗示这 2 个性状对于环境篮选表现出较高的整体趋同适应性, 体现了植物功能性状对 群落间环境变异的依赖性大于群落内种间相互作用的依赖性。
\end{abstract}

关键词 漓江; 河岸带; 功能性状; 尺度变异；性状梯度分析；咒余分析；相关性；零模型

梁士楚, 刘润红, 荣春艳, 常斌, 姜勇 (2019). 漓江河岸带植物功能性状变异与关联. 植物生态学报, 43, 16-26. DOI: 10.17521/cjpe.2018.0119

\section{Variation and correlation of plant functional traits in the riparian zone of the Lijiang River, Guilin, Southwest China}

LIANG Shi-Chu, LIU Run-Hong, RONG Chun-Yan, CHANG Bin, and JIANG Yong*

Key Laboratory of Ecology of Rare and Endangered Species and Environmental Protection, Ministry of Education, Guangxi Normal University/College of Life Science, Guangxi Normal University, Guilin, Guangxi 541006, China

\begin{abstract}
Aims Patterns of variation in plant functional traits and the correlation among them are important for understanding species coexistence and the maintenance of biodiversity. Our objectives in this study were to understand how variation and correlation of plant functional traits, at both the species and community levels, influence 1) plants adaptation to changing environments, and 2) the mechanisms of community assembly.

Methods We investigated species composition of riparian plant communities in 36 plots along the longitudinal gradient (represented by upstream, midstream, downstream) of the Lijiang River, Guilin, Southwest China. We measured three functional traits for 42 woody plant species: leaf area $(L A)$, specific leaf area (SLA), and wood density $(W D)$. For each plant community, we calculated 1) species abundance-weighted mean community trait values, and 2) species-level mean trait values. For each of these calculations, we used trait-gradient analysis to partition the three traits into alpha and beta components. We then conducted Pearson correlations to analyze the relationships among the three traits along the longitudinal gradient. Finally we tested the strength of environmental filtering using a null model that generates randomly assembled communities with species richness given by observed values.

Important findings The species abundance-weighted mean community value of $L A$ was lowest in the midstream communities, which was significantly different from that in the downstream communities. The mean community value of $W D$ for midstream and upstream communities was significantly higher than that for downstream communities. Mean community value of SLA was significantly different among the three reaches. The beta components of the three functional traits significantly differed among the three reaches and had observed values that are, on

收稿日期Received: 2018-05-20 接受日期Accepted: 2018-12-08

基金项目：国家“十二五”科技支撑计划(2012BAC16B03)和国家自然科学基金(31860124)。Supported by the National Science and Technology Pillary Program during the Twelfth Five-year Plan Period of China (2012BAC16B03), and the National Natural Science Foundation of China (31860124).

* 通信作者Corresponding author (yongjiang226@126.com)
\end{abstract}


average, lower than simulated values. However, alpha components for all three traits were not significantly different among the three reaches and had consistently lower variation than beta components. This implies that the variation in the mean community trait value across plots was greater than trait variation between species within plots. The observed and simulated values of the alpha components for both $L A$ and SLA were weakly correlated with each other within and among communities, which suggests that there are independent axes of differentiation among coexisting species. On the other hand, comparisons between observed and simulated values indicated that significantly negative correlations between $S L A$ and $W D$ were largely the result of strong environmental filters. Finally, these results imply that variation of plant functional traits is greater among communities than within communities.

Key words Lijiang River; riparian zone; functional traits; scale variation; trait-gradient analysis; RDA ordination; correlation; null model

Liang SC, Liu RH, Rong CY, Chang B, Jiang Y (2019). Variation and correlation of plant functional traits in the riparian zone of the Lijiang River, Guilin, Southwest China. Chinese Journal of Plant Ecology, 43, 16-26. DOI: 10.17521/cjpe.2018.0119

植物功能性状是指易于观测或者度量的植物特 征, 如生长型、最大高度、木材密度、比叶面积、 叶片氮磷含量、果实类型、种子大小和散布方式等 植物形态、生理和物候等属性(Cornelissen et al., 2003; 刘晓娟和马克平，2015)。它是植物在漫长的 进化和发展过程中与环境相互作用的结果, 能够客 观反映植物对外界环境的适应能力(Donovan et al., 2011), 可以作为调解器来预测环境变化对植物群 落的影响(Suding \& Goldstein, 2008)。一直以来，植 物功能性状沿环境梯度如何变化是研究植物群落构 建机制的焦点问题之一(McGill et al., 2006; 孟婷婷 等, 2007; 曹科, 2014)。但基于生态位理论的植物群 落构建理论认为不同尺度植物群落组成不仅取决于 环境因素(如气候、土壤、地形以及干扰)的作用，同 时又受到群落内生物间相互作用的影响而产生群落 物种的不同性状组成(Cornwell et al., 2006)。首先, 通过环境節滤作用塑造植物性状, 即过滤出一系列 适合特定环境条件的具有相似功能性状的物种, 导 致群落内共存物种性状的趋同性; 另一方面, 群落 内过于相似的物种发生竞争排斥, 从而减少物种间 的生态位重叠和缓解资源竞争压力, 导致了相似生 境中群落物种的性状趋异(Gewin, 2006)。因此, 功 能性状值的变化范围被认为是限制共存物种的环境 过滤(性状变化范围减小)(Cornwell et al., 2006)和竞 争导致的生态位分化共同作用的结果(性状变化范 围增大) (Stubbs \& Wilson, 2004; Grime, 2006)。那么, 如何根据性状变异格局推断环境过滤和生物竞争两 种作用力的相对重要性始终是生态学领域的一个重 大挑战。Ackerly和Cornwell (2007)提出的性状梯度 分析法, 通过将物种性状值分解为群落内 ( $\alpha$ 组分) 和
群落间 ( $\beta$ 组分)两个组分, 为解决这一问题提供了有 力的工具。如果存在明显的环境梯度, 生境篮选对 群落构建起主导作用, 可以预期不同性状的 $\beta$ 组分 应该有很强的协变关系, 不同物种 $\beta$ 组分的离散度 应该低于随机期望; 反之, 如果竞争排斥起主导作 用, 则物种在不同策略轴上分割生态位, 可以预期 不同性状的 $\alpha$ 组分仅有很弱的协变关系，不同物种 $\alpha$ 组分的离散度应该高于随机期望。因此, 研究物种 的 $\alpha 、 \beta$ 组分如何变异与关联对深入理解不同尺度上 群落构建的机制具有重要的理论意义。

河岸带是指高低水位之间的河床以及高水位之 上直至河水影响完全消失的地带 (Nilsson \& Berggren, 2000)。显然, 河岸带的主要特点在于它是 介于河流与陆地之间的过渡地带, 形成了其独特的 植被、土壤、地形地貌和水文特性(段文军等, 2014)。 如, 河溪的水文周期会随着时空变化而产生不定期 的变化。其次, 水生与陆生环境的明显不同。最后, 上、中、下游的生境也存在高度异质性, 河流在纵 向上的蜿蜒曲折, 水流对河岸的冲刷侵蚀(韩路等, 2013; 黄芗等, 2013), 也造成河流横断面呈现不同 生境类型, 这些特征决定了河岸带植物适应的独特 性, 形成了特殊的植物性状组合。如, 黄端等(2017) 研究发现漓江水陆交错带重度淹没区相比微度淹没 区, 植物叶片具有较大的最大净光合速率、叶氮含 量和叶磷含量, 植物通过提高叶片光合效率和相关 营养水平响应淹没期的深度水淹胁迫。因此, 河岸 带植物功能性状的分异规律可能与地带性植被不同, 这为研究功能性状跨多尺度变异提供了良好的研究 对象, 然而目前关于漓江河岸带植物功能性状变异 与关联的研究尚未见报道。鉴于此, 本研究选取漓 
江河岸带不同河段的植物群落作为研究对象, 采用 性状梯度法、相关性分析和零模型等方法, 分析该 区植物功能性状的变异和关联, 以期回答以下 3 个 科学问题: (1)漓江河岸带植物功能性状沿不同河段 的变化规律如何? (2)功能性状在不同尺度(群落间 和群落内)上的变化是否存在差异? (3)功能性状在 不同尺度(群落间和群落内)具有怎样的关联性? 这 些科学问题的合理解答, 不但可以揭示环境篮选与 生物相互作用在群落构建中的作用, 而且有助于我 们从功能生态学的角度来探究群落物种组成和结构 等的动态变化及其动力, 从而为漓江河岸带植被管 理和恢复等提供科学依据。

\section{1 材料和方法}

\section{1 研究区概况}

漓江处于广西壮族自治区东北部，位于珠江流 域西江水系一级支流——桂江的上游河段, 地理坐 标为 $109.75^{\circ}-110.67^{\circ} \mathrm{E}, 24.30^{\circ}-25.68^{\circ} \mathrm{N}$ 。根据降水、 地形、径流等特征, 可将漓江划分为上游、中游、 下游 3 个河段, 其中桂林水文站至源头为上游, 桂林 水文站至阳朔水文站为中游, 阳朔水文站至恭城河 口为下游(刘金荣等, 2003)。漓江流域属中亚热带湿 润季风气候区, 全年光照充足, 气候温和, 四季分 明, 雨量充沛, 雨热基本同期, 平均气温 $17.8-19.1{ }^{\circ} \mathrm{C}$, 年降水量1 814-1 941 mm, 年蒸发量 1 377-1 857 $\mathrm{mm}$, 无霜期长达 300 余天。漓江属于雨源型山区河 流, 径流为降雨所形成, 年径流量比较丰富, 但年 内和年际分布极不均匀, 汛期为3-8月, 径流占年径 流量的近 $80 \%$, 其中 5-6月占年径流量的 $40 \%$, 枯水 期为 9 月至翌年 2 月, 最枯月 1 月径流量仅占年径流 量的 $2 \%$ (段文军等, 2014)。漓江河岸带土壤类型以 山地黄壤为主, 经过江水的持续冲刷和沉积, 具有 石砾含量高、土层浅薄、土层厚度分布不均等特点 (李青山等, 2014)。漓江河岸带常见乔木有枫杨 (Pterocarya stenoptera)、乌柏(Sapium sebiferum)、朴 树(Celtis sinensis)、阴香(Cinnamomum burmannii)、 樟(Cinnamomum camphora) 等; 主要灌木有石榕树 (Ficus abelii)、牡荆(Vitex negundo var. cannabifolia)、 白饭树(Flueggea virosa)、萝芙木(Rauvolfia verticillata)、细叶水团花(Adina rubella); 主要草本有狗 牙根(Cynodon dactylon)、辣蓼(Polygonum hydropiper)、喜旱莲子草(Alternanthera philoxeroides)、竹
叶草 (Oplismenus compositus)、麦冬(Ophiopogon japonicus $) 、$ 红花酢浆草(Oxalis corymbosa)、酢浆草 (Oxalis corniculata)、葎草(Humulus scandens)、皱叶 狗尾草(Setaria plicata)等(信忠保等, 2014; 李扬等, 2015)。

\section{2 群落学调查}

经过现场踏查，于2016年9-10月和2017年7-9 月, 在漓江河岸带上游、中游、下游各建立了 12 个 大小为 $20 \mathrm{~m} \times 20 \mathrm{~m}$ 的样方, 合计 36 个样方, 各样方 的基本情况如表 1 所示。将每个样方划分成 4 个 $10 \mathrm{~m}$ $\times 10 \mathrm{~m}$ 的小样方, 对各样方进行常规的群落学调查, 内容主要包括: 对乔木层植株进行每木检尺, 记录 种名、胸径等指标; 灌木层植株记录种名、高度、 基径等指标。同时，记录各样方的土壤类型、海拔、 距河距离、砾石裸露率、砾石直径、干扰程度等生 境特征。土壤采样以 $5 \mathrm{~m} \times 5 \mathrm{~m}$ 小样方为基本单位, 采用“梅花五点法”, 在每个 $5 \mathrm{~m} \times 5 \mathrm{~m}$ 小样方的 4 个 顶点和中心位置, 清除表层枯落物和腐殖质层后, 用直径 $10 \mathrm{~cm}$ 的土钻钻取 0-20 cm 土层土样, 运用四 分法混合均匀后、放置于室内阴凉通风处自然干燥。 待风干后, 剔除其中的根系、石块、钲核及动植物 残体等杂物后倒入研钵中研细, 过不同孔径 $(0.15$ 、 $0.25 、 1.4 \mathrm{~mm}$ ) 的土壤篎, 对预处理后的土样进行土 壤 $\mathrm{pH}$ 值、有机质含量、全氮含量、速效氮含量、全 磷含量、速效磷含量、全钾含量、速效钾含量 8 个土 壤化学性质指标的测定, 测定方法参照《土壤农业 化学常规分析方法》(中国土壤学会农业化学专业委 员会, 1983)。每个土壤样品重复测定3次后取其平均 值作为本研究分析的数据。

\section{3 功能性状的选取及测定}

选取叶面积 $(L A)$ 、比叶面积 $(S L A)$ 和木材密度 $(W D) 3$ 个重要功能性状进行测量。其中, $L A\left(\mathrm{~cm}^{2}\right)$ 反 映叶片与外界环境接触面积的大小, 其会影响到植 物体与外界环境的气体、能量等交换的平衡, 一般 生在阴蔽、湿润、资源丰富地区的植物具有较大的 叶片(Craine \& Lee, 2003; Sun et al., 2006)。SLA 是新 鲜叶片面积与叶片干质量的比值 $\left(\mathrm{cm}^{2} \cdot \mathrm{g}^{-1}\right)$, 反映植 物投入单位质量的干物质所获得的捕光面积, 与潜 在相对生长速率或最大光合速率正相关, 物种具有 较大的 $S L A$ 意味着生长速率较高, 养分利用效率较 低, “防御性”资源投入较少, 叶片寿命相对较短 (Cornelissen et al., 2003)。WD是枝条干质量与体积 
表1 漓江河岸带不同河段植物群落样方基本概况

Table 1 Basic information of the sampled plots in the riparian zone along the longitudinal gradient of the Lijiang River

\begin{tabular}{|c|c|c|c|c|c|c|}
\hline $\begin{array}{l}\text { 河段 } \\
\text { Reach }\end{array}$ & $\begin{array}{l}\text { 样方数 } \\
\text { Plot number }\end{array}$ & $\begin{array}{c}\text { 海拔 } \\
\text { Elevation }(\mathrm{m})\end{array}$ & $\begin{array}{c}\text { 温度 } \\
\text { Temperature }\left({ }^{\circ} \mathrm{C}\right)\end{array}$ & $\begin{array}{c}\text { 降水量 } \\
\text { Precipitation }(\mathrm{mm})\end{array}$ & $\begin{array}{l}\text { 干扰强度 } \\
\text { Disturbance intensity }\end{array}$ & $\begin{array}{l}\text { 群落类型 } \\
\text { Community type }\end{array}$ \\
\hline \multirow{5}{*}{$\begin{array}{l}\text { 上游 } \\
\text { Upstream }\end{array}$} & 3 & 154 & 24.7 & 1941 & 轻度 & 枫杨一石榕树群落 \\
\hline & & & & & Light & Pterocarya stenoptera-Ficus abelii communities \\
\hline & 3 & 148 & 23.0 & 1941 & 轻度 & 枫杨+朴树一夢芙木群落 \\
\hline & & & & & Light & $\begin{array}{l}\text { Pterocarya stenoptera }+ \text { Celtis sinensis- } \\
\text { Rauvolfia verticillata } \text { communities }\end{array}$ \\
\hline & 6 & 144 & 23.7 & 1941 & $\begin{array}{l}\text { 轻度 } \\
\text { Light }\end{array}$ & $\begin{array}{l}\text { 枫杨+阴香一石榕树群落 } \\
\text { Pterocarya stenoptera + Cinnamomum bur- } \\
\text { mannii-Ficus abelii communities }\end{array}$ \\
\hline \multirow[t]{3}{*}{$\begin{array}{l}\text { 中游 } \\
\text { Midstream }\end{array}$} & 4 & 138 & 26.0 & 1900 & $\begin{array}{l}\text { 重度 } \\
\text { High }\end{array}$ & $\begin{array}{l}\text { 枫杨十乌柏一细叶水团花群落 Pterocarya } \\
\text { stenoptera }+ \text { Sapium sebiferum-Adina rubella } \\
\text { communities }\end{array}$ \\
\hline & 5 & 134 & 25.0 & 1900 & $\begin{array}{l}\text { 重度 } \\
\text { High }\end{array}$ & $\begin{array}{l}\text { 阴香群落 Cinnamomum burmannii commu- } \\
\text { nities }\end{array}$ \\
\hline & 3 & 104 & 24.0 & 1900 & $\begin{array}{l}\text { 重度 } \\
\text { High }\end{array}$ & $\begin{array}{l}\text { 枫杨一夢芙木群落Pterocarya stenoptera- } \\
\text { Rauvolfia verticillata communities }\end{array}$ \\
\hline \multirow[t]{2}{*}{$\begin{array}{l}\text { 下游 } \\
\text { Downstream }\end{array}$} & 8 & 111 & 30.1 & 1900 & $\begin{array}{l}\text { 中度 } \\
\text { Middle }\end{array}$ & $\begin{array}{l}\text { 乌柏+朴树一牡荆群落Sapium sebiferum + } \\
\text { Celtis sinensis-Vitex negundo var. cannabifo- } \\
\text { lia } \text { communities }\end{array}$ \\
\hline & 4 & 105 & 26.8 & 1900 & $\begin{array}{l}\text { 中度 } \\
\text { Middle }\end{array}$ & $\begin{array}{l}\text { 乌柏一木槿群落 Sapium sebiferum + Hibis- } \\
\text { cus syriacus } \text { communities }\end{array}$ \\
\hline
\end{tabular}

的比值 $\left(\mathrm{g} \cdot \mathrm{cm}^{-3}\right)$, 其与植物坚向生长的结构性支撑 力、植物的寿命、树干的防御功能(如病虫害、可食 性和物理性防御等)及碳储量有关, 可以反映植物 的结构性投入和生长死亡的权衡关系( 卜文圣等, 2013)。

测定样方内所有胸径 $\geqslant 1 \mathrm{~cm}$ 的木本植物植株个 体的功能性状(包括乔、灌木和木质藤本)。在野外 群落学调查过程中, 每个植物个体选取冠层顶端 3 片完全展开的健康成熟的阳生叶及3根长 $10 \mathrm{~cm}$ 左右, 直径为 $1-2 \mathrm{~cm}$ 的非当年生枝条放入密封袋内, 置于 阴暗环境下并迅速带回实验室进行叶和茎功能性状 测定。对于叶性状的测定, 利用叶面积仪 (Yaxin-1241, 北京雅欣理仪科技有限公司, 北京)扫 描每片叶片的叶面积, 然后将叶片样品编号后置于 $80{ }^{\circ} \mathrm{C}$ 烘箱中烘干至恒质量后, 称量并记录叶干质 量。对于木材密度的测定, 本文利用测定的枝条密 度代替木材密度，将枝条削去表面的树皮后，使用 电子天平测定其鲜质量, 接着用量筒排水法测定枝 条的体积, 然后将其编号并置于 $80{ }^{\circ} \mathrm{C}$ 烘箱中烘干 至恒质量后，测定枝条干质量。

\section{4 统计与分析}

\subsection{1 几余分析}

首先，采用单因素方差分析对不同河段间的 8 个土壤化学性质 $(\mathrm{pH}$ 值、全氮含量、有机质含量、
速效氮含量、全磷含量、速效磷含量、全钾含量、 速效钾含量)指标进行统计检验，根据分析结果排 除部分不显著因子; 再结合土壤类型、海拔、距河 距离、砾石裸露率、干扰程度、气候因子(温度和降 水量) 构建样地 $\times$ 环境变量矩阵。其次, 基于样地 $\times$ 多 度矩阵、样地 $\times$ 性状矩阵、样地 $\times$ 环境变量矩阵, 利 用R 3.4.3 软件中 Vegan包对矩阵进行圥余分析 (RDA)。

\subsection{2 层级方差分解}

运用R 3.4.3 软件 “Ime”包中限制最大似然法 (REML), 利用广义线性模型和“varcomp” 函数对种 内、种间、群落间、河段间和河段内与河段间植物 功能性状的分异大小分别进行方差分解。

\subsection{3 性状梯度分析}

根据Ackerly和Cornwell (2007)提出的性状梯度 分析法, 将植物群落(数量为 $n$ )内的物种(数量为 $s$ ) 按 照该物种多度 $\left(\alpha_{i j}\right)$ 加权计算出其群落 $j$ 的平均性状值 $\left(p_{j}\right)$, 再根据群落平均性状值由小到大的顺序进行 排序, 构建性状梯度, 然后利用该性状梯度将物种 性状值 $\left(t_{i}\right)$ 累加降解为 $\beta$ 和 $\alpha$ 两个组分。其中, $\beta$ 组分 $\left(\beta_{i}\right)$ 为物种 $i$ 在该性状梯度上所处的位置, 体现物种 $i$ 在 不同群落间的变异, 反映物种 $i$ 对群落间环境变化 的响应; 而 $\alpha$ 组分 $\left(\alpha_{i}\right)$ 是某一物种 $i$ 性状值 $\left(t_{i}\right)$ 与其所 处群落平均性状值 $\left(\beta_{i}\right)$ 的差值, 体现了群落内物种 $i$ 
与其共存物种的性状值差异, 反映了对同一群落内 种间交互作用等非生物环境适应策略的差异。性状 梯度分析法主要计算公式如下

$$
\begin{aligned}
& p_{j}=\sum_{i=1}^{S} a_{i j} t_{i j} / \sum_{i=1}^{S} a_{i j} \\
& t_{i}=\sum_{j=1}^{n} a_{i j} t_{i j} / \sum_{j=1}^{n} a_{i j} \\
& \beta_{\mathrm{i}}=\sum_{j=1}^{n} p_{j} a_{i j} / \sum_{j=1}^{n} a_{i j} \\
& \alpha_{i}=t_{i}-\beta_{i}
\end{aligned}
$$

式中, $p_{j}$ 为群落平均性状值, $t_{i}$ 为物种平均性状值, $a_{i j}$ 为物种 $i$ 在群落 $j$ 中的多度或权重, $t_{i j}$ 为物种 $i$ 在群落 $j$ 中的性状值, $n$ 表示调查的群落总数, $s$ 表示调查出现 的物种总数。本研究中 $n=36, s=42$, 每个群落有 3-25个物种。性状梯度分析之前对不满足正态分布 的数据(除木材密度外)进行 $\lg$ 转换。利用单因素方差 分析检验漓江河岸带不同河段间的功能性状指标 (物种平均性状、 $\alpha$ 组分、 $\beta$ 组分和群落平均性状) 是 否存在显著差异。如果存在显著差异, 则采用多重 比较方法检测两两河段之间的功能性状值是否存在 差异。然后分别对功能性状指标进行Pearson相关性 分析，相关性大小用Student's $t$ 检验。

为了直观地解释性状梯度分析法, 现以常绿乔 木阴香和落叶灌木牡荆的 $S L A$ 为例来介绍 $\alpha$ 和 $\beta$ 两个 组分的生态学意义(图1)。根据上述公式计算得出阴 香的 $\beta$ 组分相对较小 $\left(\lg \beta_{i}=2.37\right)$, 位于由群落平均 $S L A$ 构建的性状梯度的下半部分, 说明阴香常见于 平均 $S L A$ 较低的群落中且阴香的 $S L A$ 小于群落的平 均值 $\left(\lg \alpha_{i}=-0.28\right)$, 表明与其共存物种相比, 其 SLA 相对较低。相反, 牡荆的 $\beta$ 组分相对较大 $\left(\lg \beta_{i}=2.53\right)$, 位于性状梯度的上半部分, 说明告荆常见于高 $S L A$ 的群落中且牡荆的 $S L A$ 大于群落的平均值 $\left(\lg \alpha_{i}=\right.$ 0.09 ), 说明与其共存物种相比, 其 $S L A$ 相对较高。

\subsection{4 零模型分析}

采用零模型的方法计算随机期望下的不同性状 $\alpha / \beta$ 组分协变强度和不同物种 $\beta$ 组分的离散度。其中, $\alpha / \beta$ 组分协变强度的零模型是根据Ackerly和Cornwell (2007)自带的源程序进行计算; $\beta$ 组分的离散度零模 型是基于Jung等(2010)提出的环境篮检验。根据实

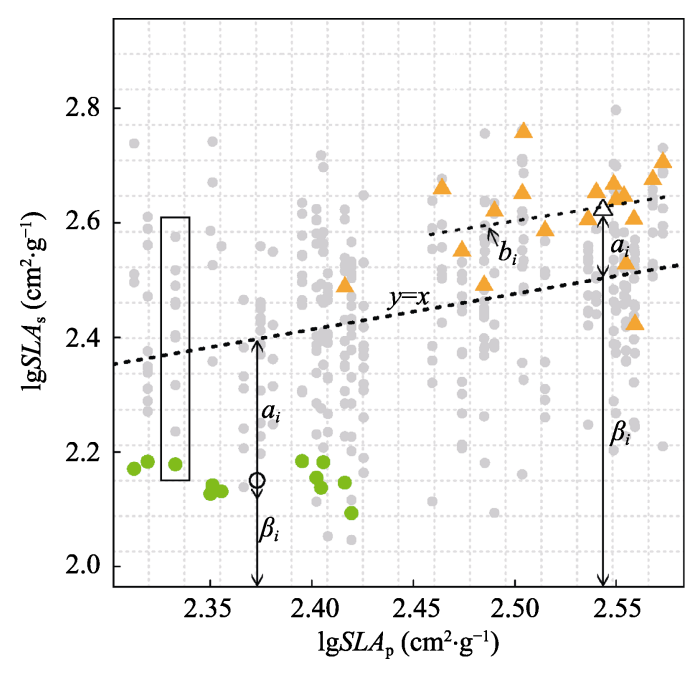

图 1 漓江河岸带植物阴香和牡荆物种平均比叶面积 $\left(\lg S L A_{\mathrm{s}}\right)$ 和群落平均比叶面积 $\left(\lg S L A_{\mathrm{p}}\right)$ 散点图。灰色圆点代表 群落中的各个物种, 绿色实心圆点和橙色三角形分别表示 阴香和牡荆, 在某 $p_{j}$ 处被矩形框起来的一列点表示群落 $j$ 内 所有共生物种。每个物种空心的图形点对应的横坐标代表该 物种在样地中所处的平均位置(即该物种性状的 $\beta$ 组分 $\beta_{i}$ ), 纵 坐标是物种的平均性状值 $t_{i}$, 二者的差值即空心的图形点到 $y=x$ 的距离 (因为 $\alpha_{i}=t_{i}-\beta_{i}$ ) 就是物种功能性状的 $\alpha$ 组分 $\alpha_{i}$, 代 表群落中物种 $i$ 平均比叶面积与共存物种平均比叶面积的差 值, $b_{i}$ 为物种 $i$ 平均比叶面积 $\left(t_{i}\right)$ 对群落平均 $S L A\left(p_{j}\right)$ 的回归直 线的斜率, 反映物种平均 SLA沿群落平均性状梯度在种内的 变化大小。

Fig. 1 The scatterplot between species mean specific leaf area (i.e. $\lg S L A_{\mathrm{s},} \mathrm{cm}^{2} \cdot \mathrm{g}^{-1}$ ) vs. plot mean specific leaf area (i.e. $\lg S L A_{\mathrm{p}}, \mathrm{cm}^{2} \cdot \mathrm{g}^{-1}$ ) between Cinnamomum burmannii and Vitex negundo in the riparian zone of the Lijiang River. Each grey point represents a species in a specific plot; the green solid points and the orange solid triangles represent Cinnamomum burmannii and Vitex negundo respectively, and a column of grey points in a black rectangle represent all the species within community. For each species, the abscissa values of the large open point show the mean position of occupied plots (i.e., the beta component of the species trait value, $\beta_{i}$ ), while the ordinate values of the solid symbols are their mean species trait value $\left(t_{i}\right)$. The difference between $\beta_{i}$ and $t_{i}$, or the distance from the $y=x$ line is $\alpha_{i}$ (because $\alpha_{i}=t_{i}-\beta_{i}$ ). Regression line shows abundance-weighted least squares regression of species trait values relative to plot mean trait values, with slope $b_{i} . b_{i}$ is the slope of each species' s regression line of species mean trait values $\left(t_{i}\right)$ relative to plot mean trait values $\left(p_{j}\right)$, it reflects the intraspecific variation of the species mean specific leaf area along a gradient defined by community-level mean trait values.

际观测建立一个河岸带不同河段间的植物物种库, 然后随机抽取与之不同河段间相同数目的物种, 进 行不放回式999次随机抽样, 计算其功能性状的分 布范围。与随机模拟群落相比, 如存在环境篎效应, 群落中的某些物种被过滤出, 导致落入植物功能性 状值的范围变小。根据随机模拟获取不同河段间的 功能性状 $\beta$ 组分分布范围的次数, 计算其次数的 $5 \%$ 和 $95 \%$ 的置信区间，若在置信区间之内，表明不同 
河段间植物功能性状 $\beta$ 组分与随机群落无显著差异; 若次数有 $5 \%$ 小于或 $95 \%$ 大于不同河段间的实际功 能性状 $\beta$ 组分分布范围, 表明不同河段间的实际植 物功能性状显著大于或小于随机群落。

\section{2 结果}

\section{1 不同河段间植物功能性状与环境因子的RDA} 排序

RDA分析结果如图2和表2所示: 上中下游河段 被群落平均功能性状 $(L A, S L A$ 和 $W D)$ 显著分开, 同 时展示了在每个梯度上环境因子对功能性状的显著 影响。 $W D$ 和 $S L A$ 主要与RDA第一排序轴相关, 处于 第一轴相对位置; $L A$ 主要与第二轴相关。 $L A$ 主要受 土壤有机质 $(\mathrm{SOM})$ 含量、干扰强度 $(D I)$ 、土壤 $\mathrm{pH}$ 值、 土壤全氮(TN)含量、降水量(Pre)显著正影响; $S L A$ 主 要受海拔 $(E L e)$ 的显著正影响; $W D$ 主要受土壤有效 氮(AN) 含量、温度 $(T e m)$ 、河段 $(R e a)$ 、距离河岸距 离 $(D i s)$ 的显著正影响。

\section{2 不同河段植物功能性状的变化规律}

统计分析如表3所示: 对于物种性状, $W D$ 是上 游与下游差异性显著。对于 $\beta$ 组分, $L A 、 S L A$ 和 $W D 3$ 个性状在两两河段中均存在显著性差异, 而在 $\alpha$ 组 分中 3 个性状在两两河段中均无显著性差异。对于群 落性状, $L A$ 是中游和下游差异显著; $S L A$ 是两两河段 中均差异性显著; $W D$ 是上游和中游显著大于下游。

\section{3 样方平均性状、物种功能性状及其 $\boldsymbol{\alpha}$ 组分、 $\boldsymbol{\beta}$} 组分的相关性

3 个功能性状相关性如图3所示: 在物种水平上, $S L A$ 和 $W D$ 之间呈显著负相关关系(图3A); 在样方尺 度上, $L A$ 和 $S L A$ 分别与 $W D$ 呈极显著负相关关系 $(p<$
0.01 )(图3D); 在 $\beta$ 组分上, $L A$ 和 $S L A$ 分别与 $W D$ 在真 实观测值和随机模拟值均呈现极显著负相关关系 $(p<0.01)$ (图3B); 在 $\alpha$ 组分上, $S L A$ 与 $W D$ 在真实观测 值和随机模拟值均呈现极显著负相关关系 $(p<0.01)$ (图3C)。

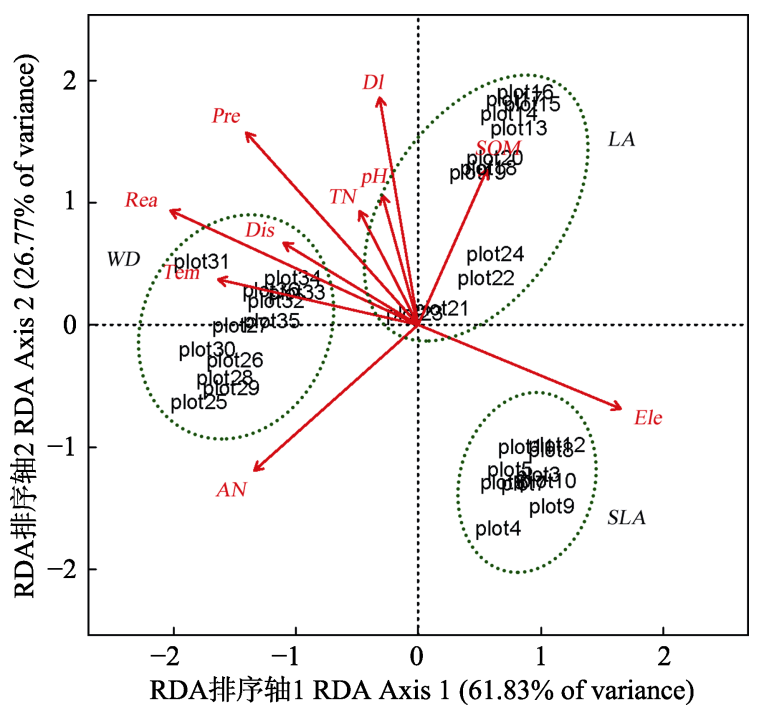

图2 漓江河岸带植物群落水平功能性状与环境因子的冗余 分析(RDA)排序图。 $A N$, 有效氮含量; $D I$, 干扰强度; $D i s$, 距 离河岸距离; Ele, 海拔; $L A$, 叶面积; $p H$, 土壤 $\mathrm{pH}$ 值; Pre, 降水量; Rea, 河段; SLA, 比叶面积; SOM, 有机质含量; Tem, 温度; $T N$, 全氮含量; $W D$, 木材密度。plot 1-12, 上游; plot 12-24, 中游; plot 25-36, 下游。

Fig. 2 Redundancy analysis (RDA) ordination diagram showing the relationships between the three abundance weighted functional traits and 10 selected environmental factors of the riparian plant of the Lijiang River. $A N$, soil available nitrogen; $D I$, disturbance intensity; Dis, distance; Ele, elevation; $L A$, leaf area; $p H$, soil $\mathrm{pH}$ value; Pre, precipitation; Rea, reach; SLA, specific leaf area; SOM, soil organic matter content; Tem, temperature; $T N$, soil total nitrogen content; $W D$, wood density. plot 1-12, upstream; plot 12-24, midstream; plot 25-36, downstream.

表2＼cjkstart漓江河岸带群落水平功能性状与环境因子咒余分析(RDA)中的前2轴环境变量解释方差和显著性检验

Table 2 The explained variance of environmental factors and their significant analysis in the first two axes in redundancy analysis (RDA) ordination

\begin{tabular}{|c|c|c|c|c|}
\hline 环境因子 Environmental factor & $\mathrm{RDA}_{1}$ & $\mathrm{RDA}_{2}$ & $\overline{R^{2}}$ & $p$ \\
\hline 有机质 Soil organic matter $\left(\mathrm{g} \cdot \mathrm{kg}^{-1}\right)$ & 0.40 & 0.91 & 0.34 & $0.002^{* *}$ \\
\hline 全氮 Soil total nitrogen $\left(\mathrm{g} \cdot \mathrm{kg}^{-1}\right)$ & -0.45 & 0.88 & 0.19 & $0.032^{*}$ \\
\hline 有效氮 Soil available nitrogen $\left(\mathrm{mg} \cdot \mathrm{kg}^{-1}\right)$ & -0.74 & -0.66 & 0.57 & $0.001^{* * *}$ \\
\hline $\mathrm{pH}$ & -0.26 & 0.96 & 0.21 & $0.015^{*}$ \\
\hline 干扰强度Disturbance intensity & -0.16 & 0.98 & 0.63 & $0.001^{* * *}$ \\
\hline 距离河岸距离 Distance (m) & -0.85 & 0.51 & 0.29 & $0.004^{* *}$ \\
\hline 降水量 Precipitation (mm) & -0.66 & 0.74 & 0.79 & $0.001^{* * *}$ \\
\hline 温度 Temperature $\left({ }^{\circ} \mathrm{C}\right)$ & -0.97 & 0.22 & 0.50 & $0.001^{* * *}$ \\
\hline 海拔 Elevation (m) & 0.92 & -0.38 & 0.57 & $0.001^{* * *}$ \\
\hline 河段 Reach & -0.90 & 0.41 & 0.88 & $0.001^{* * *}$ \\
\hline
\end{tabular}

$*, p<0.05 ; * *, p<0.01 ; * * *, p<0.001$ 
表3 漓江不同河段功能性状参数值(平均值土标准偏差)

Table 3 Statistics of the three plant functional traits across the three reaches of Lijiang River (mean $\pm S D$ )

\begin{tabular}{|c|c|c|c|c|c|}
\hline \multirow[t]{2}{*}{ 河段 Reach } & \multirow{2}{*}{$\begin{array}{l}\text { 功能性状 } \\
\text { Functional trait }\end{array}$} & \multicolumn{4}{|c|}{ 性状参数 Functional trait parameter } \\
\hline & & 物种性状值 $t_{i}$ & $\beta$ 组分 $\beta_{i}$ & $\alpha$ 组分 $\alpha_{i}$ & 群落性状值 $p_{j}$ \\
\hline \multirow{3}{*}{$\begin{array}{l}\text { 上游 } \\
\text { Upstream }\end{array}$} & 叶面积 $L A\left(\mathrm{~cm}^{2}\right)$ & $1.30 \pm 0.35^{\mathrm{a}}$ & $1.36 \pm 0.08^{\mathrm{a}}$ & $-0.06 \pm 0.34^{\mathrm{a}}$ & $1.34 \pm 0.14^{\mathrm{ab}}$ \\
\hline & 比叶面积 $S L A\left(\mathrm{~cm}^{2} \cdot \mathrm{g}^{-1}\right)$ & $2.40 \pm 0.13^{\mathrm{a}}$ & $2.40 \pm 0.02^{\mathrm{a}}$ & $-0.00 \pm 0.13^{\mathrm{a}}$ & $2.39 \pm 0.03^{\mathrm{a}}$ \\
\hline & 木材密度 $W D\left(\mathrm{~g} \cdot \mathrm{cm}^{-3}\right)$ & $0.47 \pm 0.10^{\mathrm{a}}$ & $0.44 \pm 0.02^{\mathrm{a}}$ & $0.03 \pm 0.10^{\mathrm{a}}$ & $0.44 \pm 0.04^{\mathrm{a}}$ \\
\hline \multirow{3}{*}{$\begin{array}{l}\text { 中游 } \\
\text { Midstream }\end{array}$} & 叶面积 $L A\left(\mathrm{~cm}^{2}\right)$ & $1.16 \pm 0.32^{\mathrm{a}}$ & $1.24 \pm 0.08^{\mathrm{b}}$ & $-0.08 \pm 0.27^{\mathrm{a}}$ & $1.26 \pm 0.10^{\mathrm{a}}$ \\
\hline & 比叶面积 $S L A\left(\mathrm{~cm}^{2} \cdot \mathrm{g}^{-1}\right)$ & $2.47 \pm 0.21^{\mathrm{a}}$ & $2.47 \pm 0.06^{\mathrm{b}}$ & $0.01 \pm 0.21^{\mathrm{a}}$ & $2.46 \pm 0.07^{\mathrm{b}}$ \\
\hline & 木材密度 $W D\left(\mathrm{~g} \cdot \mathrm{cm}^{-3}\right)$ & $0.42 \pm 0.12^{\mathrm{ab}}$ & $0.47 \pm 0.01^{\mathrm{b}}$ & $-0.04 \pm 0.12^{\mathrm{a}}$ & $0.47 \pm 0.02^{\mathrm{a}}$ \\
\hline \multirow{3}{*}{$\begin{array}{l}\text { 下游 } \\
\text { Downstream }\end{array}$} & 叶面积 $L A\left(\mathrm{~cm}^{2}\right)$ & $1.24 \pm 0.32^{\mathrm{a}}$ & $1.45 \pm 0.05^{\mathrm{c}}$ & $-0.20 \pm 0.30^{\mathrm{a}}$ & $1.46 \pm 0.07^{\mathrm{b}}$ \\
\hline & 比叶面积 $S L A\left(\mathrm{~cm}^{2} \cdot \mathrm{g}^{-1}\right)$ & $2.48 \pm 0.13^{\mathrm{a}}$ & $2.53 \pm 0.02^{\mathrm{c}}$ & $-0.06 \pm 0.12^{\mathrm{a}}$ & $2.55 \pm 0.03^{\mathrm{c}}$ \\
\hline & 木材密度 $W D\left(\mathrm{~g} \cdot \mathrm{cm}^{-3}\right)$ & $0.36 \pm 0.10^{\mathrm{b}}$ & $0.34 \pm 0.01^{\mathrm{c}}$ & $0.02 \pm 0.10^{\mathrm{a}}$ & $0.34 \pm 0.02^{\mathrm{b}}$ \\
\hline
\end{tabular}

同一功能性状同列不同字母表示差异显著 $(p<0.05)$

$L A$, leaf area; $S L A$, specific leaf area; $W D$, wood density; $t_{i}$, species trait values; $\alpha_{i}$, alpha components; $\beta_{i}$, beta components; $p_{j}$ plot means. Different lowercase letters for the same functional trait in each column mean significant difference at the level of $p=0.05$.
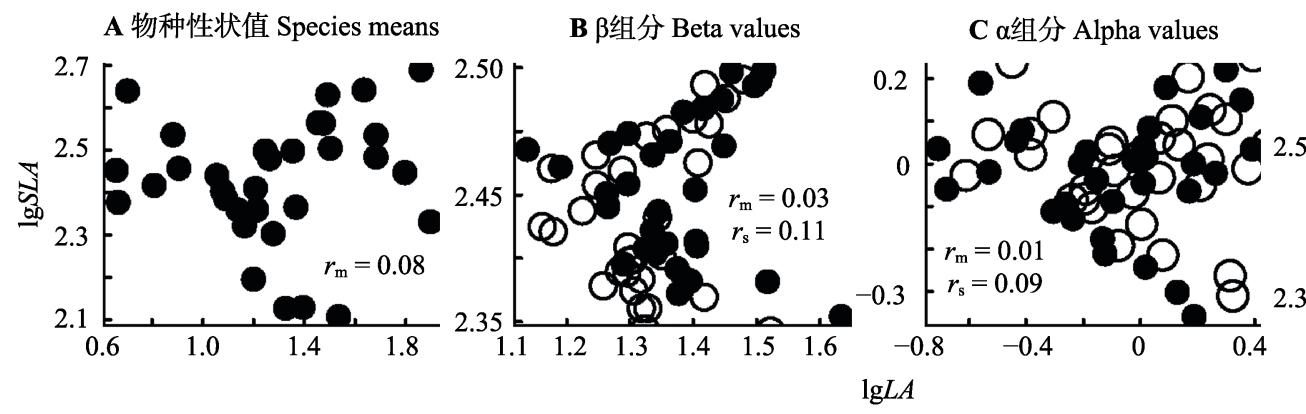

D 样方平均性状值 Plot means
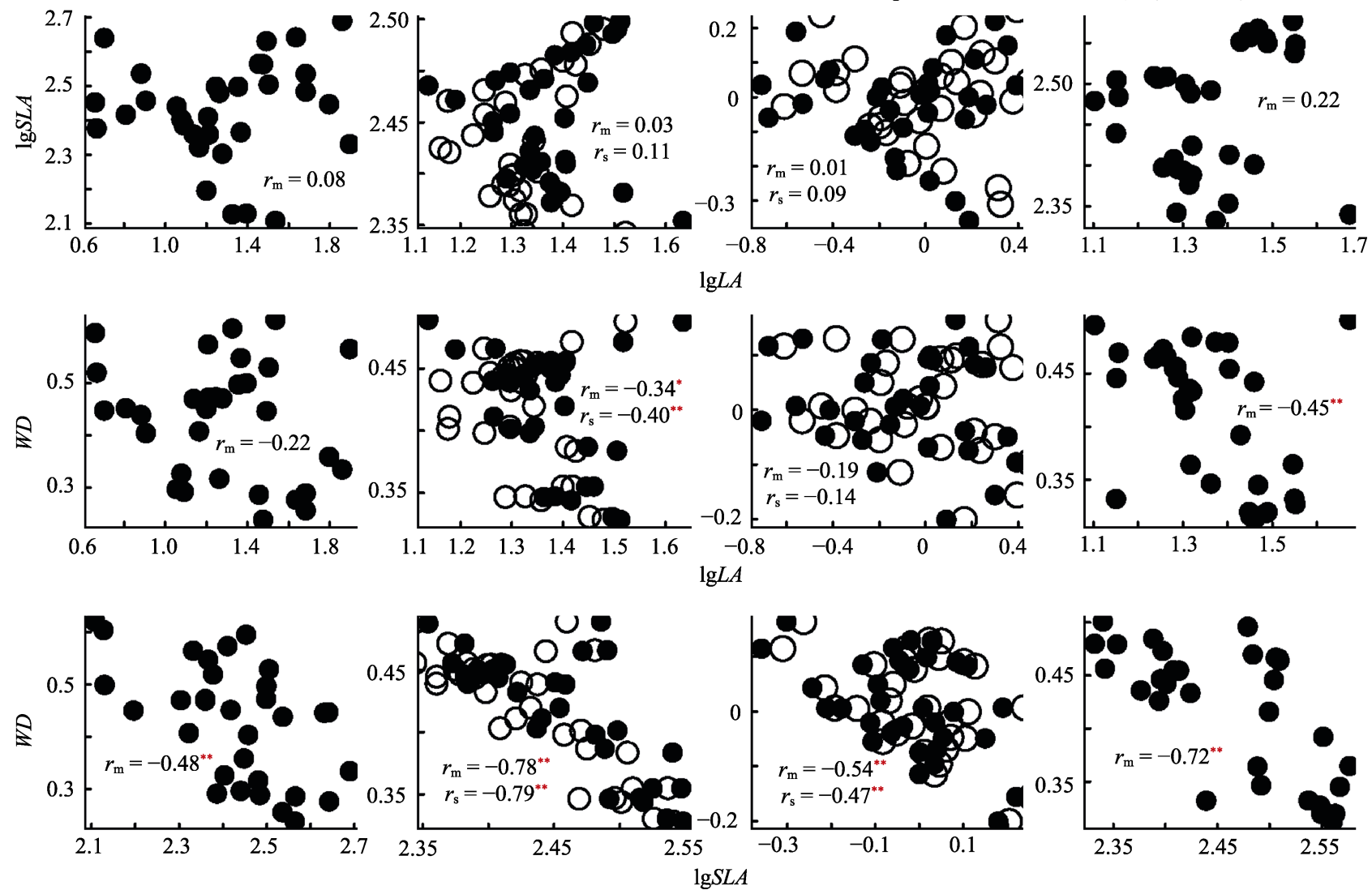

图3 漓江河岸带植物叶面积 $(L A)$ 、比叶面积 $(S L A)$ 和木材密度 $(W D)$ 在物种性状值 $(\mathbf{A}) 、 \beta$ 组分 $(\mathbf{B}) 、 \alpha$ 组分 $(\mathbf{C}) 、$ 样方平均性状值 (D)水平上的相关性散点图以及Pearson相关系数 $(r)$ 。黑色实心点和 $r_{\mathrm{m}}$ 分别代表实际测量数据和实测相关系数, 黑色圆圈和 $r_{\mathrm{s}}$ 分别代表零模型随机模拟 999 次数据和模拟相关系数。*, $p<0.05 ; * *, p<0.01$ 。

Fig. 3 Scatterplots showing relationships between leaf area $(L A)$, specific leaf area $(S L A)$ and wood density (WD) for species trait values (A), beta components (B), alpha components $(\mathbf{C})$, and plot mean trait values $(\mathbf{D})$ of the riparian plant of the Lijiang River. The Pearson correlation coefficient $(r)$ of these relationships are shown in each figure. Black solid dots and $r_{\mathrm{m}}$ respectively represent observed values and observed correlation coefficient; black open circles and $r_{\mathrm{s}}$ respectively represent random simulation values and simulation coefficient. *, $p<0.05 ; * *, p<0.01$.

2.4 不同河段植物功能性状 $\beta$ 组分的分布范围与零 模型的比较分析

检测发现 3 个功能性状 $\beta$ 组分实际观测值均小于
零模型，表明了功能性状 $\beta$ 组分分布范围在 3 个不同 河段受到环境的筛选作用且与零模型差异是否显著 随河段的变化而变化。 $L A$ 实际观测值在下游与零模

www.plant-ecology.com 
型差异显著 $(p<0.05) ; S L A$ 实际观测值在上游和下 游均与零模型差异显著 $(p<0.05)$; $W D$ 实际观测值 在上、中、下游均与零模型差异显著 $(p<0.05$ )(图4)。

\section{5 不同水平上的植物功能性状的方差分异}

三个功能性状值 $(L A 、 S L A$ 和 $W D)$ 均体现群落水 平性状变异最小, 其次是河段, 再次是种内, 种间 性状变异最大, 即种间>种内>河段间>群落间(图 $5 \mathrm{~A})$ 。SLA和 $W D$ 在河段内和河段间的变异规律较一 致, 即河段间>河段内; 但是 $L A$ 相反, 表现为河段 内>河段间(图5B)。

\section{3 讨论}

\section{1 群落平均性状 $\boldsymbol{\alpha} 、 \boldsymbol{\beta}$ 组分间沿河岸带不同河段变 化规律}

河岸带植物群落的生态特征受空间位置、干扰 强度、气候、地形、土壤养分以及淹水的深度、持 续时间和频率等环境因子的显著影响, 呈现随着环 境梯度变化其梯度变化同样明显的特征(Vannote et al., 1980; 孙荣等, 2010)。本研究通过RDA排序结果 展示了上中下游河段间不同环境因子组合(包括干 扰强度强弱、海拔梯度、土壤养分含量等)对功能性
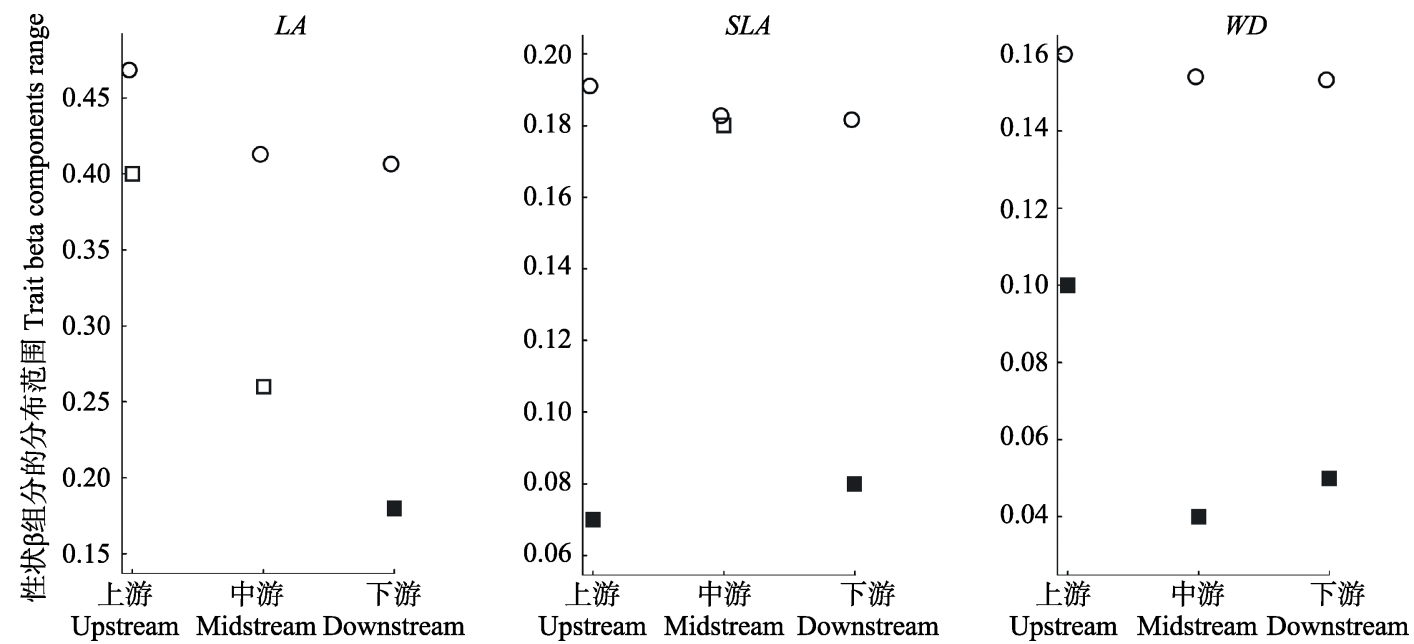

图4 漓江不同河段植物功能性状 $\beta$ 组分的分布范围与零模型比较。圆圈代表零模型预测值; 空心正方形代表实际观测值; 实 心正方形代表实际观测值与零模型存在显著差异 $(p<0.05)$ 。

Fig. 4 Difference on beta components ranges in the observed and simulated values of the three functional traits at the three reaches in Lijiang River. Circles and squares represent simulated and observed values respectively. Filled squares indicate that the observed values differ significantly from the simulated values.
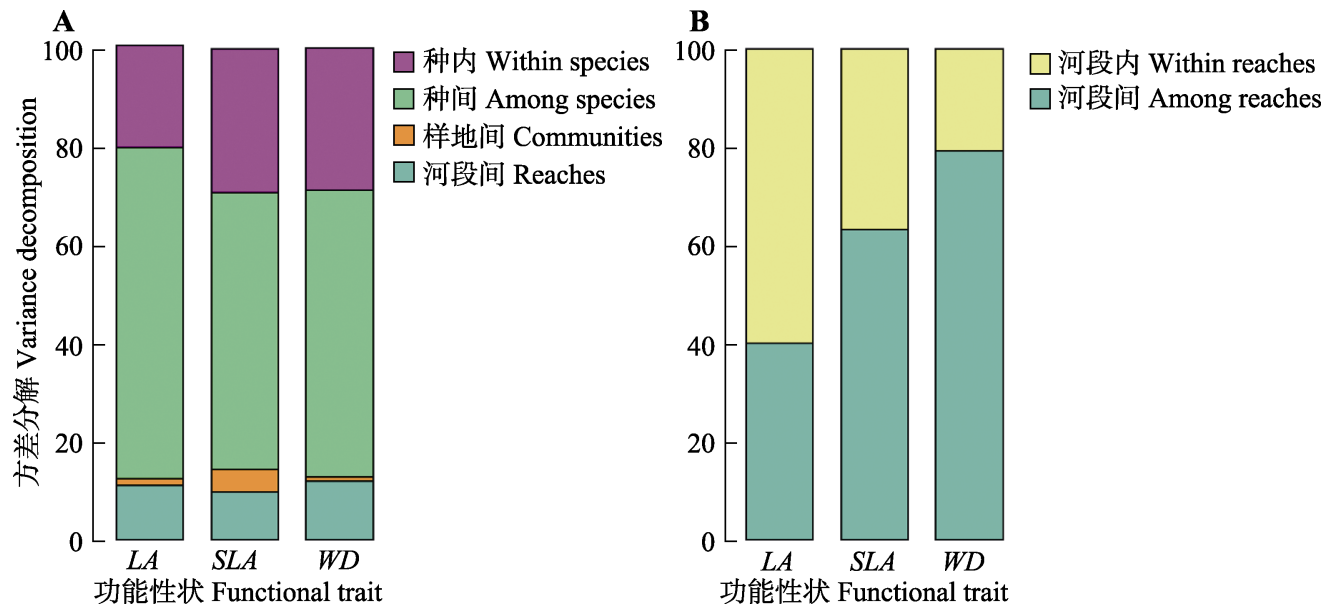

图5 漓江河岸带植物种内、种间、群落间与河段间(A)及河段内和河段间(B)植物功能性状的方差分解。 $L A$, 叶面积; SLA, 比 叶面积; $W D$, 木材密度。

Fig. 5 Partitioning of the variance in plant functional traits explained by four scales (i.e. within-specie, among-species, communities and reaches) (A) and by two scales (i.e. within and among reaches) (B) of the riparian plant of the Lijiang River. $L A$, leaf area; $S L A$, specific leaf area; $W D$, wood density. 
状( $L A 、 S L A$ 和 $W D)$ 产生了显著影响。结合图5B中的 $S L A$ 和 $W D$ 在河段间>河段内的性状变异规律进一步 印证了不同河段间的环境梯度主导功能性状变异。 图5B中 $L A$ 功能性状分布变异呈现相反的变化规律, 即河段内>河段间, 但二者变异比例相差不大, 出 现这样的变异规律是很出乎意料的, 同时也是值得 深思的。这可能与采样误差及性状的选择有关。因 此, 我们希望通过改进实验方案和尝试其他植物功 能性状包括数值型性状和类别性状获取进一步验 证。针对不同梯度下生境的高度异质性, 发现漓江 河岸带群落平均性状 $L A 、 S L A$ 和 $W D$ 在上、中、下游 河段之间均存在着显著的差异(表2; 图2), 表明植 物群落为了适应河岸带不同河段的生境条件采取了 不同的生态策略。例如, 群落平均 SLA 随着纵向梯度 变化逐渐增大且差异性显著, 暗示了物种面对生境 的变化而采取的适应性策略不同。即从上游物种光 合速率低、叶寿命长且防御能力较强, 常绿物种比 例较高逐渐转变为提高生长速率而降低养分利用效 率的落叶灌木物种占主导。群落平均 $W D$ 是中、上 游高于下游, 表现出与 SLA相反的性状梯度, 根据 植物经济谱学说, 恶劣环境下选择慢收益型策略, 即物种具有 $S L A$ 更小 $W D$ 更高的性状特征。结合RDA 排序结果和表 1 发现上流河段的环境资源比较贯乏, 这与上游生境特征相关, 即海拔和地势相对较高, 坡度较陡峭, 土壤层较薄, 砾石含量较高, 取样点 距离河水距离较近, 导致淋溶作用强烈, 土壤中的 营养成分流失严重, 尽管干扰最轻。下游相对地势 平坦, 土壤层较厚, 土壤中养分含量随着地表水由 上游流向下游地段积累, 土壤养分含量相对较高且 温度适宜, 采样地点距离河水距离较远及干扰适度 (中度干扰), 对比来讲下流河段生境较好。群落平均 $L A$ 是中游小于下游且差异显著, 主要受干扰强度影 响, 其次受土壤有机质含量、土壤 $\mathrm{pH}$ 值、土壤全氮 含量、降水量等环境因子显著影响(图2; 表2)。实地 调查发现中游距离市区最近, 旅游业盛行, 岸边过 度艮荒, 人为干扰严重, 导致了中游物种种类偏少 且叶面积大的物种比例较低, 结合图 $5 \mathrm{~A}$ 发现植物功 能性状 $(L A 、 S L A$ 和 $W D)$ 均为种间变异贡献最大, 说 明不同河段间的物种组成差异显著, 印证了面对干 扰严重的恶劣生境 $L A$ 采取了慢收益保守型策略。此 外, 本研究发现 3 个功能性状的 $\beta$ 组分在河岸带不同 河段间的统计检验均差异性显著, 且河岸带不同河
段的 3 个植物功能性状 $\beta$ 组分的观测值均小于随机 模拟的零模型分布(图3), 表明了功能性状 $\beta$ 组分分 布范围在河岸带不同河段受到环境篎作用的限制作 用较为明显; 但 $\alpha$ 组分均差异性不显著(表3)且在河 岸带不同河段 3 个功能性状 $\alpha$ 组分的分布范围均小 于 $\beta$ 组分, 说明在河岸带不同河段的群落构建过程 中环境因素的影响要大于群落内种间的相互作用, 则性状梯度很大程度上取决于环境梯度。

\section{2 植物功能性状及其 $\boldsymbol{\alpha} 、 \boldsymbol{\beta}$ 组分间的相关性}

植物在其漫长的历史进化和发展过程中, 受物 理、生理等因素的综合作用和影响，功能性状之间 往往会呈现出一定的协变和权衡关系(Wright et al., 2007; Hu et al., 2015)。Fonseca等(2000)和Ackerly等 (2002)发现 $L A$ 与 $S L A$ 在全球和区域尺度上均呈显著 正相关关系。但是, Shipley (1995)和Grubb (1998)发 现同一群落内植物的 $L A$ 与 $S L A$ 之间呈现不相关或负 相关关系。这表明在地理尺度上 $L A$ 与 $S L A$ 均受某种 选择因素(一般而言是气候因素)的限制, 但是在局 域尺度上的适应可能会导致这两者从属于不同生态 策略维度。本研究发现, 漓江河岸带木本植物 $L A$ 与 $S L A$ 在物种功能性状值、样方功能性状值及其 $\beta$ 组分 值相关性很弱 $(r$ 分别为 $0.08 、 0.22$ 和 0.03$)$, 尤其是 $\alpha$ 组分值几乎不存在相关关系 $(r=0.01)$ 。这一结果基 本与习新强等(2011)对黔中喀斯特山区植物功能性 状变异与关联的研究结果一致。SLA与 $L A$ 在物种尺 度没有显著相关性, 这主要是由 $\alpha_{S L A}$ 与 $\alpha_{L A}$ 之间没有 显著相关关系造成的。此外, 群落水平和 $\beta$ 组分二者 均相关性不显著。Baraloto等(2010)分析热带雨林的 叶和茎经济型谱发现, $L A$ 和 $S L A$ 呈正交, 即两个性 状属于不同的策略维度。由此可见, 在局域尺度上 $L A$ 和 $S L A$ 各自代表了植物在不同生态策略上的维度, 在本研究中得到了很好的佐证。

$W D$ 与 $L A$ 两性状在物种水平和 $\beta$ 组分相关性存 在显著差异 $(p<0.01)$, 但 $\alpha$ 组分相关性较弱且零模 型的变化趋势与观测值变化一致。造成这一格局的 原因可能是, 群落环境梯度导致优势物种从喜阳的 落叶灌木物种 (大 $L A$, 小 $W D$ ) 逐渐变为耐阴的常绿 乔木(小 $L A$, 大 $W D$ ), 反映在性状上就是这两个性状 的 $\beta$ 组分呈明显的负相关关系。而在群落内部, 不同 的物种产生不同的性状组合来分化生态位 (Cornwell et al., 2006; Kraft et al., 2008; Kunstler et $a l .$, 2016)形成大致的竞争均衡, 导致二者的 $\alpha$ 组分 
协变关系很低(图3C)。此外, 本研究中 $W D$ 与 $S L A$ 在 各水平上均呈现出显著负相关关系且零模型检测与 此变化一致, 暗示了植物功能性状SLA和 $W D$ 对于环 境篮选表现出较高的整体趋同适应性, 导致二者的 $\alpha 、 \beta$ 组分均具有很强的协变关系, 而不同物种 $\beta$ 组分 的离散度很大(表2)。这进一步反映了漓江上中下游 的主导性环境梯度对于群落性状变异的强限制作 用。

致谢 感谢2017年广西高校优秀教师出国项目、广 西硕士研究生创新项目(YCSW2017079)和2017年 度自治区级大学生创新创业训练计划项目 (201710602236和201710602247)资助。

\section{参考文献}

Ackerly DD, Cornwell WK (2007). A trait-based approach to community assembly: Partitioning of species trait values into within- and among-community components. Ecology Letters, 10, 135-145.

Ackerly DD, Knight CA, Weiss SB, Barton K, Starmer KP (2002). Leaf size, specific leaf area and microhabitat distribution of chaparral woody plants: Contrasting patterns in species level and community level analyses. Oecologia, 130, 449-457.

Agricultural Chemistry Committee of Soil Science Society of China (1983). Conventional Methods for the Agricultural Chemical Analysis of Soil. Science Press, Beijing. [中国土 壤学会农业化学专业委员会 (1983). 土壤农业化学常 规分析方法. 科学出版社, 北京.]

Baraloto C, Timothy Paine CE, Poorter L (2010). Decoupled leaf and stem economics in rain forest trees. Ecology Letters, 13, 1338-1347.

Bu WS, Zang RG, Ding Y, Zhang JY, Ruan YZ (2013). Relationships between plant functional traits at the community level and environmental factors during succession in a tropical lowland rainforest on Hainan Island, South China. Biodiversity Science, 21，278-287. [卜文圣, 藏润国, 丁 易, 张俊艳, 阮云泽 (2013). 海南岛热带低地雨林群落 水平植物功能性状与环境因子相关性随演替阶段的变 化. 生物多样性, 21, 278-287.]

Cao K (2014). The Phylogeny Signal of Functional Traits and Their Relationship between Each Other and Effects on Community Structure. Master degree dissertation, Zhejiang Normal University, Jinhua, Zhejiang. [曹科 (2014). 古田 山植物功能性状的系统发育信号、不同性状之间的关系 及其对群落结构的影响. 硕士学位论文, 浙江师范大 学, 浙江金华.]

Cornelissen JHC, Lavorel S, Garnier E, Díaz S, Buchmann N, Gurvich DE, Reich PB, Steege H, Morgan HD, Heijden MGA, Pausas JG, Poorter H (2003). A handbook of protocols for standardized and easy measurement of plant functional traits worldwide. Australian Journal of Botany, 51, 335-380.

Cornwell WK, Schwilk DW, Ackerly DD (2006). A trait-based test for habitat filtering: Convex hull volume. Ecology, 87, 1465-1471.

Craine JM, Lee WG (2003). Covariation in leaf and root traits for native and non-native grasses along an altitudinal gradient in New Zealand. Oecologia, 134, 471-478.

Donovan LA, Maherali H, Caruso CM, Huber H, Kroom HD (2011). The evolution of the worldwide leaf economics spectrum. Trends in Ecology and Evolution, 26, 88-95.

Duan WJ, Wang JY, Zhang LJ, Li HF, Huang HQ (2014). Characteristics of precipitation in Lijiang River Basin during 1960 2010. Journal of China Hydrology, 34(5), 88-93. [段文军, 王金叶, 张立杰, 李海防, 黄华乾 (2014). 1960 2010年漓江流域降水变化特征研究. 水 文, 34(5), 88-93.]

Fonseca CR, Overton JM, Collins B, Westoby M (2000). Shifts in trait-combinations along rainfall and phosphorus gradients. Journal of Ecology, 88, 964-977.

Gewin V (2006). Beyond neutrality-Ecology finds its niche. PLOS Biology, 4, 1306-1310.

Grime JP (2006). Trait convergence and trait divergence in herbaceous plant communities: Mechanisms and consequences. Journal of Vegetation Science, 17, 255-260.

Grubb P (1998). A reassessment of the strategies of plants which cope with shortages of resources. Perspectives in Plant Ecology, Evolution and Systematics, 1, 3-31.

Han L, Wang HZ, Yu J (2013). Research progress and prospects on riparian zone ecology. Ecology and Environmental Sciences, 22，879-886. [韩路, 王海珍, 于军 (2013). 河岸带生态学研究进展与展望. 生态环境学报, 22, 879-886.]

Hu YK, Pan X, Liu GF, Li WB, Dai WH, Tang SL, Zhang YL, Xiao T, Chen LY, Xiong W, Zhou MY, Song YB, Dong M (2015). Novel evidence for within-species leaf economics spectrum at multiple spatial scales. Frontiers in Plant Science, 6, 901. DOI: 10.3389/fpls.2015.00901.

Huang D, Wang DM, Ren Y, Qin YB, Wu LC (2017). Responses of leaf traits to submergence stress and analysis of the economic spectrum of plant species in an aquaticterrestrial ecotone, the Li River. Acta Ecologica Sinica, 37, 750-759. [黄端, 王冬梅, 任远, 覃云斌, 吴林川 (2017). 漓江水陆交错带植物叶性状对水淹胁迫的响应 及经济谱分析. 生态学报, 37, 750-759. ]

Huang Y, Que XX, Li CY (2013). Study on landscape ecological restoration technology of land/inland water ecotones along Li River. Journal of Southern Agriculture, 44, 1700-1704. [黄莹, 烕欣欣, 李彩云 (2013). 漓江沿岸 水陆交错带景观调查与生态修复技术. 南方农业学报, 44, 1700-1704.]

Jung V, Violle C, Mondy C, Hoffmann L, Muller S (2010). Intraspecific variability and trait-based community assembly. Journal of Ecology, 98, 1134-1140. 
Kraft NJB, Valencia R, Ackerly DD (2008). Functional traits and niche-based tree community, assembly in an Amazonian forest. Science, 322, 580-582.

Kunstler G, Falster D, Coomes DA, Hui F, Kooyman RM, Laughlin DC, Poorter L, Vanderwel M, Vieilledent G, Wright SJ, Aiba M, Baraloto C, Caspersen J, Cornelissen JHC, Gourlet-Fleury S, Hanewinkel M, Herault B, Kattge J, Kurokawa H, Onoda Y, Peñuelas J, Poorter H, Uriarte M, Richardson S, Ruiz-Benito P, Sun I-F, Ståhl G, Swenson NG, Thompson J, Westerlund B, Wirth C, Zavala MA, Zeng H, Zimmerman JK, Zimmermann NE, Westoby M (2016). Plant functional traits have globally consistent effects on competition. Nature, 529, 204-207.

Li QS, Wang DM, Xin ZB, Li Y, Ren Y (2014). Root distribution in typical sites of Lijiang ecotone and their relationship to soil properties. Acta Ecologica Sinica, 34, 2003-2011. [李青山, 王冬梅, 信忠保, 李扬, 任远 (2014). 漓江水陆交错带典型立地根系分布与土壤性质 的关系. 生态学报, 34, 2003-2011.]

Li Y, Wang DM, Xin ZB, Wang J, Ren Y, Li QS (2015). Plant diversity and soil characteristics of different inundation zones in an aquatic-terrestrial ecotone, Li River. Acta Ecologica Sinica, 35, 5121-5130. [李扬, 王冬梅, 信忠 保，王晶，任远，李青山 (2015). 漓江水陆交错带不同 淹没区植物多样性与土壤特征. 生态学报, 35 , 5121-5130.]

Liu JR, Feng H, Yu XL, Song GJ, Ye Q (2003). A preliminary discussion on the historic change of the name for the Lijiang River system. Carsologica Sinica, 22(1), 77-83. [刘金荣, 冯红, 俞秀兰, 宋桂金, 叶青 (2003). 历史上 漓江(桂江)水系名称的变化浅议. 中国岩溶, 22(1), 77-83.]

Liu XJ, Ma KP (2015). Plant functional traits-Concepts, applications and future directions. Scientia Sinica (Vitae), 45, 325-339. [刘晓娟, 马克平 (2015). 植物功能性状研究 进展. 中国科学: 生命科学, 45, 325-339.]

McGill BJ, Enquist BJ, Weiher E, Westoby M (2006). Rebuilding community ecology from functional traits. Trends in Ecology \& Evolution, 21, 178-185.

Meng TT, Ni J, Wang GH (2007). Plant functional traits, environments and ecosystem functioning. Journal of Plant Ecology (Chinese Version), 31, 150-165. [孟婷婷, 倪健, 王国宏 (2007). 植物功能性状与环境和生态系统功能. 植物生态学报, 31, 150-165.]

Nilsson C, Berggren K (2000). Alterations of riparian ecosystems caused by river regulation. Bioscience, 50, 783-792.
Shipley B (1995). Structured interspecific determinants of specific leaf area in 34 species of herbaceous angiosperms. Functional Ecology, 9, 312-319.

Stubbs WJ, Wilson JB (2004). Evidence for limiting similarity in a sand dune community. Journal of Ecology, 92, $557-567$.

Suding KN, Goldstein LJ (2008). Testing the Holy Grail framework: Using functional traits to predict ecosystem change. New Phytologist, 180, 559-562.

Sun R, Yuan XZ, Chen ZL, Zhang YW, Liu H (2010). Patterns of plant community species richness in the fluctuating water level zone along the Pengxihe River of the Three Gorges Reservoir. Research of Environmental Sciences, 23，1382-1389. [孙荣，袁兴中，陈忠礼，张跃伟，刘红 (2010). 三峡水库澎溪河消落带植物群落物种丰富度格 局. 环境科学研究, 23, 1382-1389.]

Sun SC, Jin DM, Shi PL (2006). The leaf size-twig size spectrum of temperate woody species along an altitudinal gradient: An invariant allometric scaling relationship. Annals of Botany, 97, 97-107.

Vannote RL, Minshall GW, Cumins KW, Sedell JR, Cushing CE (1980). The river continuum concept. Canadian Journal of Fisheries and Aquatic Sciences, 37, 130-137.

Wright IJ, Ackerly DD, Bongers F, Harms KE, IbarraManriquez G, Martinez-Ramos M, Mazer SJ, Muller-Landau HC, Paz H, Pitman NCA, Poorter L, Silman MR, Vriesendorp CF, Webb CO, Westoby M, Wright SJ (2007). Relationships among ecologically important dimensions of plant trait variation in seven Neotropical forests. Annals of Botany, 99, 1003-1015.

Xi XQ, Zhao YJ, Liu YG, Wang X, Gao XM (2011). Variation and correlation of plant functional traits in karst area of central Guizhou Province, China. Chinese Journal of Plant Ecology, 35，1000-1008. [习新强, 赵玉杰, 刘玉国, 王 欣, 高贤明 (2011). 黔中喀斯特山区植物功能性状的变 异与关联. 植物生态学报, 35, 1000-1008.]

Xin ZB, Xiao YL, Wang DM, Li Y, Ren Y, Li QS (2014). Spatial patterns of riparian vegetations and its optimization in Lijiang River: An intensive tourism karsts river in the southern subtropical China. Ecological Science, 33, 631-641. [信忠保, 肖玉玲, 王冬梅, 李扬, 任远, 李青 山 (2014). 广西桂林漓江河岸带植被配置类型与退化 机制研究. 生态科学, 33, 631-641.]

责任编委: 储诚进 责任编辑: 李 敏 